\title{
Buoyancy Induced Heat Transfer and Fluid Flow inside a Prismatic Cavity
}

\author{
A. Walid ${ }^{1}$ and O. Ahmed ${ }^{2}$ \\ ${ }^{I}$ LESTE, ENIM, Monastir, Tunisia \\ ${ }^{2}$ FSG, Gafsa, Tunisia \\ Email:aich_walid@yahoo.fr \\ (Received July 9, 2009; accepted November 5, 2009)
}

\begin{abstract}
This paper deals with a numerical simulation of natural convection flows in a prismatic cavity. This configuration represents solar energy collectors, conventional attic spaces of greenhouses and buildings with pitched roofs. The third dimension of the cavity is considered long enough for the flow to be considered 2D. The base is submitted to a uniform heat flux, the two top inclined walls are symmetrically cooled and the two vertical walls are assumed to be perfect thermal insulators. The aim of the study is to examine the thermal exchange by natural convection and effects of buoyancy forces on flow structure. The study provides useful information on the flow structure sensitivity to the governing parameters, the Rayleigh number ( $\mathrm{Ra}$ ) and the aspect ratio of the cavity. The hydrodynamic and thermal fields, the local Nusselt number, the temperature profile at the bottom and at the center of the cavity are investigated for a large range of Ra. The effect of the aspect ratio is examined for different values of Ra. Based on the authors' knowledge, no previous results on natural convection in this geometry exist.
\end{abstract}

Keywords: Rayleigh number, Nusselt number, natural convection, prismatic cavity, heat transfer

\section{NOMENCLATURE}

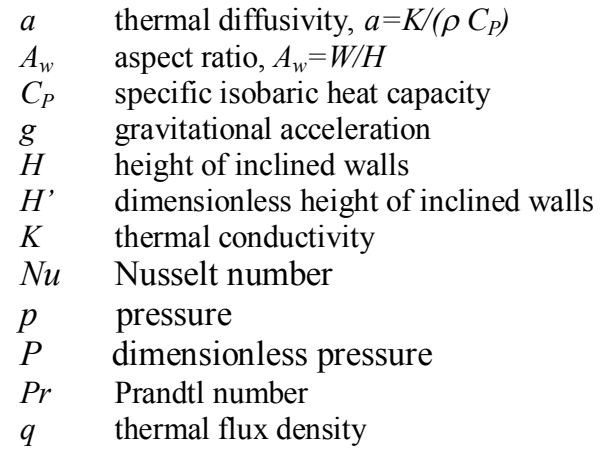

\section{INTRODUCTION}

Natural convection heat transfer and fluid flow in enclosed spaces has been studied extensively in recent years in response to energy-related applications, such as thermal insulation of buildings using air gaps, solar energy collectors, furnaces and fire control in buildings and so on. The enclosures encountered in these applications are highly diverse in their geometrical configuration and the most investigated enclosures include the annulus between horizontal cylinders, the spherical annulus, the hollow horizontal cylinder, the

$\begin{array}{ll}R a & \text { Rayleigh number, } R a=g . \beta . q . H^{4} /(K . a . v) \\ U, V & \text { dimensionless velocity components in the } \mathrm{X} \\ & \text { and Y directions } \\ W & \begin{array}{l}\text { height of vertical walls } \\ \text { horizontal and vertical dimensionless } \\ \text { coordinates }\end{array} \\ \alpha & \text { inclination angle of roofs, } \alpha=60^{\circ} \\ \beta & \text { coefficient of volumetric thermal expansion } \\ \rho & \text { fluid density } \\ v & \text { kinematic viscosity } \\ \theta & \text { dimensionless temperature }\end{array}$

closed rectangular cavity and the closed triangular cavity.

A review of the literature on natural convection in isosceles triangular cavities shows that this configuration was the object of numerous experimental and numerical studies. Earlier, the flow and temperature patterns, local wall heat fluxes and mean heat flux rates were measured experimentally by Flack $(1979,1980)$ in isosceles triangular cavities with three different aspect ratios. The cavities, filled with air, were heated/cooled from the base and cooled/ heated from the inclined 
walls covering a wide range of Grashof numbers. Asan and Namli (2000) conducted a numerical study of laminar natural convection in a pitched roof of triangular cross-section considering an adiabatic midplane wall condition in their numerical procedure. Only summertime conditions were considered over wide ranges of both $\mathrm{Ra}$ and the height-based aspect ratio. Their results showed that most of the heat exchange takes place near the intersection of the active walls. In another study (2001), they examined the laminar natural convection heat transfer in triangular-shaped roofs with different inclination angles and $\mathrm{Ra}$ values in winter day. They indicated that both aspect ratio and $\mathrm{Ra}$ affect the temperature and flow field. They also found that heat transfer decreases with the increasing of aspect ratio. The finite-element method was used by Holtzman et al. (2000) to model the complete isosceles triangular cavity without claiming cavity symmetry. A heated base and symmetrically-cooled inclined walls were considered as thermal boundary conditions for various aspect ratios and Grashof numbers. The performed experiment consisted in a flow visualization study to validate the existence of symmetry-breaking bifurcations in one cavity of fixed aspect ratio. This anomalous bifurcation phenomenon was intensified by gradually increasing the Grashof number. The main conclusion reached was that, for identical isosceles triangular cavities engaging symmetrical and nonsymmetrical assumptions, the differences in terms of mean Nusselt number were around $5 \%$. Ridouane and Campo (2005) generated experimentallybased correlations for the reliable characterization of the center plane temperature and the mean convective coefficient in isosceles triangular cavities filled with air. The experimental data were gathered from various sources for various aspect ratios and Grashof numbers. Omri et al. (2005) studied laminar natural convection in a triangular cavity with isothermal upper sidewalls and with a uniform continuous heat flux at the bottom. The study showed that the flow structure and the heat transfer are sensitive to the cavity shape and to Ra. An optimum tilt angle was determined corresponding to a minimum of the Nusselt number and for a maximum of the temperature at the bottom center. Many recirculation zones can occur making homogeneous the thermal field in the core of the cavity. Hajri et al. (2007) studied double-diffusive natural convection in a triangular cavity. The main conclusion drawn in their paper was that the buoyancy ratio and the Lewis number values have a profound influence on the thermal, concentration and dynamic fields. Results show that, for the small values of the buoyancy ratio, there is little increase in the heat and mass transfer over that due to conduction. For higher values, the convective mode dominates.

In the study of Varol et al. (2007), buoyancy-induced natural convection is investigated with a numerical technique in Gambrel roofs. The geometry was adapted to both winter day conditions (the bottom is heated while top is cooled $=$ case I) and summer day conditions (the bottom is cooled and inclined top wall is heated $=$ case II). They indicated that for a higher value of $\mathrm{Ra}$, namely $\mathrm{Ra}=4.105$, Nusselt number value increases. They also found that heat transfer is better in the winterlike boundary conditions than in the summerlike ones due to the mechanism of natural convection. In another study (2007), they examined natural convection in triangular enclosures with protruding isothermal heater. To obtain better heat removal from the heater, a higher aspect ratio must be chosen and the heater must be located to the center of the bottom. Hakan et al. (2007) examined steady natural convection heat transfer and flow field inside the shed roof with and without eave in summer day boundary conditions. The study showed that heat transfer increases with the increasing of Ra. However, due to conduction dominant regime, heat transfer becomes constant for lower values of Ra. Multiple circulation cells were obtained at the highest Ra. They also found that a decrease on eave length increases the heat transfer from the inclined wall to bottom. General observation shows that heat transfer is increased with the increase in the aspect ratio. Conduction heat transfer becomes dominant at the smallest aspect ratio for all eave lengths. Koca et al. (2007) investigated the effects of Prandtl number on natural convection heat transfer and fluid flow in a partially-heated triangular enclosure. The main conclusion drawn in this paper was that heat transfer increases with the increasing of heater length and Ra. They also found that higher heat transfer is obtained when the heater is located near the right corner of the triangular enclosure and, for all cases of heater length, a decrease of Prandtl number decreases the heat transfer. In the work of Karyakin (1989) natural convection in horizontal prismatic enclosures of arbitrary cross-section was investigated. It was found that the maximum values of the stream function and Nusselt number may accomplish damping oscillations about their stationary values.

The present paper's interest lies in studying the natural convection flow in a prismatic cavity with a bottom submitted to a uniform heat flux, two top inclined walls symmetrically-cooled and two vertical walls assumed to be adiabatic. The work has been motivated by the heat transfer problem associated with roof-type solar still and various other engineering structures. The paramount aim has been to obtain the various heat and flow parameters for such enclosures as described above. Results are presented for the steady laminar-flow regime; all the fluid properties are constant except for the density variation which was determined according to the Boussinesq approximation. Velocity-pressure formulation was applied without pressure correction. The entire physical domain is taken into consideration for the computations and no symmetry plane is assumed. This step is necessary for the present problem because, as demonstrated experimentally by Holtzman et al. (2000) for the laminar regime analysis, a pitchfork bifurcation occurs at a critical Grashof number above which the symmetric solutions become unstable and finite perturbations and asymmetric solutions are instead obtained.

\section{Analysis ANd Numerical Method}

Figure 1 indicates the schematic diagram for the used configuration and geometrical details. The model considered here is a symmetrical room submitted to different boundary conditions. 


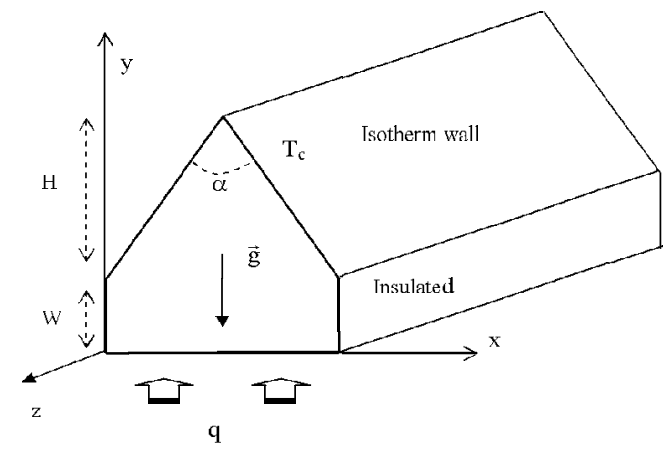

Fig. 1. Schematic of an air-filled prismatic cavity

An enclosure is composed by the juxtaposition of an upper prismatic space and a lower rectangular cavity. The bottom is exposed to a uniform heat flux q while the inclined walls are maintained at a constant temperature TC and the vertical walls are insulated. Using the primitive formulation $(\mathrm{U}, \mathrm{V}, \mathrm{P})$, the governing equations for two-dimensional, laminar incompressible buoyancy-induced flows with Boussinesq approximation and constant fluid properties in a nondimensional velocity-pressure form are:

$$
\begin{aligned}
& \frac{\partial U}{\partial X}+\frac{\partial V}{\partial Y}=0 \\
& U \frac{\partial U}{\partial X}+V \frac{\partial U}{\partial Y}=-\frac{\partial P}{\partial X}+\left[\frac{\partial^{2} U}{\partial X^{2}}+\frac{\partial^{2} U}{\partial Y^{2}}\right] \\
& U \frac{\partial V}{\partial X}+V \frac{\partial V}{\partial Y}=-\frac{\partial P}{\partial Y}+\left[\frac{\partial^{2} V}{\partial X^{2}}+\frac{\partial^{2} V}{\partial Y^{2}}\right]+\frac{R a}{\operatorname{Pr}} \theta \\
& U \frac{\partial \theta}{\partial X}+V \frac{\partial \theta}{\partial Y}=\frac{1}{\operatorname{Pr}}\left[\frac{\partial^{2} \theta}{\partial X^{2}}+\frac{\partial^{2} \theta}{\partial Y^{2}}\right]
\end{aligned}
$$

where $U$ and $V$ are, respectively, the velocity components in the $X$ and $Y$ direction; $P$ is the dimensionless pressure and $\theta$ is the dimensionless temperature.

In the generated set, the temperature is normalized as: $\theta=\frac{K \cdot\left(T-T_{C}\right)}{\mathrm{q} \cdot \mathrm{H}}$

Distances, velocity components and pressure are normalized by reference respectively to $H, \frac{v}{H}$ and $\frac{p \cdot H^{2}}{\rho v^{2}}$. The dimensionless height of the triangular part is therefore equal to unity $\left(H^{\prime}=1\right)$ and the dimensionless width of the bottom is:

$$
L=\frac{2}{\tan (\alpha)}
$$

where $\mathrm{q}$ is the value of the thermal flux at the bottom; $\alpha=60^{\circ}$ is the inclination angle of roof and $v$ is the kinematical viscosity.

A Control Volume Finite Elements Method (Omri 2000; Baliga 1978) is used in this computation. The domain of interest is divided in triangular elements and a polygonal volume is constructed around each node by joining the element's centre with the middle of its sides. The set of governing equations is integrated over the control volume with use of an exponential interpolation in the mean flow direction and a linear interpolation in the transversal direction inside the finite element. The SIMPLEC algorithm is used for the treatment of pressure-velocity coupling. The set of algebraic equations is solved using successive under relaxation (SUR) technique and 0.1 is taken as under relaxation parameter.

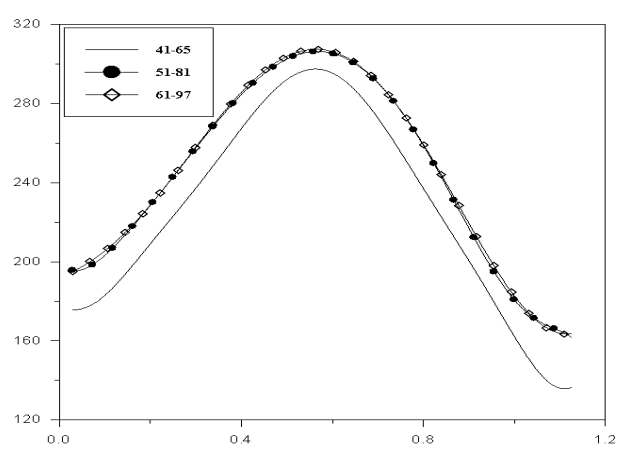

Fig. 2. Local Nusselt profile over the bottom surface for different numbers of meshes at $\mathrm{Ra}=10^{6}$

The convergence of the local Nusselt number at the heated surface with grid refinement is shown in Fig. 2 at $\mathrm{Ra}=10^{6}$. It is observed that, for an aspect ratio $A_{w}=0.25$, grid independence is achieved with a $81 \times 51$ grid beyond which there is insignificant change in $\mathrm{Nu}$.

For an aspect ratio $A_{w}=0.5$, a proportionately larger number of grid in the y-direction is used while keeping the number of grids in the $\mathrm{x}$-direction fixed at 81 . Solutions were assumed to converge when the following convergence criterion was satisfied for every variable at every point in the solution Domain.

$\left|\frac{\varphi_{\text {new }}-\varphi_{\text {old }}}{\varphi_{\max }}\right| \leq 10^{-4}$

where $\varphi$ represents $U, V, P$ and $\theta$.

The object of this paper is to report results relevant to steady natural convection in a prismatic cavity for the range $10^{3} \leq R a \leq 10^{6}$. The aspect ratio $A_{w}=W / H$ takes two values 0.25 and 0.5 .

This study is a first part of a research in which we want to understand the $2 \mathrm{D}$ dynamics and we have already proceeded to compute 3D flows.

\subsection{The Non-Dimensional Boundary Conditions}

The solution must satisfy dimensionless boundary conditions which are as follows:

- At the cover walls: $U=0, V=0$ and $\theta=0$.

- At the vertical walls: $U=0, V=0$ and $\frac{d \theta}{d n}=0$. 
- At the heated horizontal bottom, an external dimensionless thermal flux density $\frac{d \theta}{d Y}=-1$ is considered with $U=0, V=0$. The boundary condition $\frac{d \theta}{d Y}=-1$ at the bottom wall arises as a consequence of constant heat flux q boundary condition.

The transported energy across the inclined walls is expressed in terms of local and mean Nusselt numbers. The local Nusselt number can be obtained from gradients of temperatures according to the following relationship:

$$
N u_{X}=-\frac{d \theta}{d n}
$$

where $n$ is the outward drawn normal of the surface.

\subsection{Validation}

To validate the numerical analysis, this code is used in the same geometry, with the same boundary conditions used in Volker et al. (1989). This geometry is an equilateral triangular cavity heated from below and cooled at the inclined walls. The profile of the local $\mathrm{Nu}$ at the bottom in the present study and in Volker et al. (1989) is compared for $\mathrm{Ra}=10^{5}$ and satisfactory agreement was observed as shown in Fig. 3. The same code was tested against the results obtained by Tzeng et al. (2005) by comparing the local Nusselt number for $\mathrm{Ra}=2772$ with right-angle triangular enclosure. Excellent agreement was observed as reported in Fig. 4.

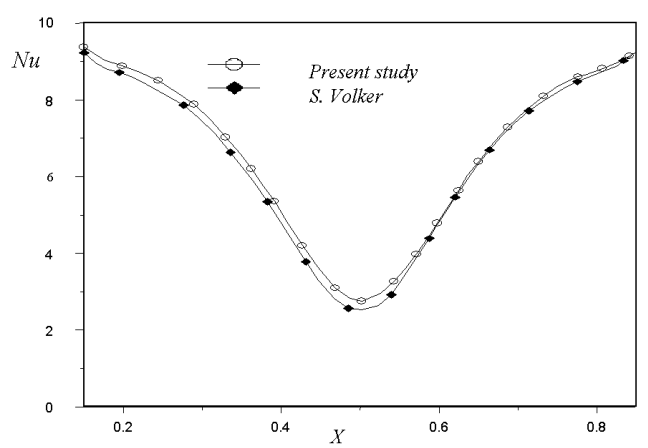

Fig. 3. Comparison of results of local Nusselt number on the bottom wall of a triangular cavity

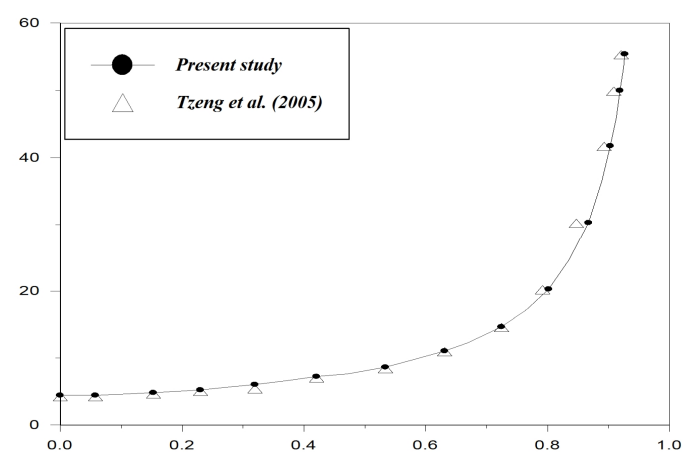

Fig. 4. Comparison of results of local Nusselt number for right-angle triangular enclosure

\section{Results AND Discussion}

\subsection{Rayleigh Number Effect}

\subsubsection{Dynamic Field}

A numerical study was performed to analyze the natural convection heat transfer in a prismatic cavity with different values of Ra. The results shown in Fig. 5 are investigated for particular aspect ratio $A_{w}=0.25$. Prandtl number is taken as 0.71 which corresponds to air. Obviously, the streamlines patterns point out that the flow loses the symmetrical structure for higher Ra values. It can be observed that two recirculation cells grow in size by increasing Ra. The left cell rotates in the anticlockwise direction and the other cell rotates in the clockwise orientation. The streamlines become tight at the mid-plane indicating that the warmed fluid is well-accelerated when buoyancy effects are stronger. This is demonstrated by Fig. 6 which gives the vertical velocity component profile and shows that the fluid is pushed upward in the central part of the cavity and is more accelerated at high Ra values.

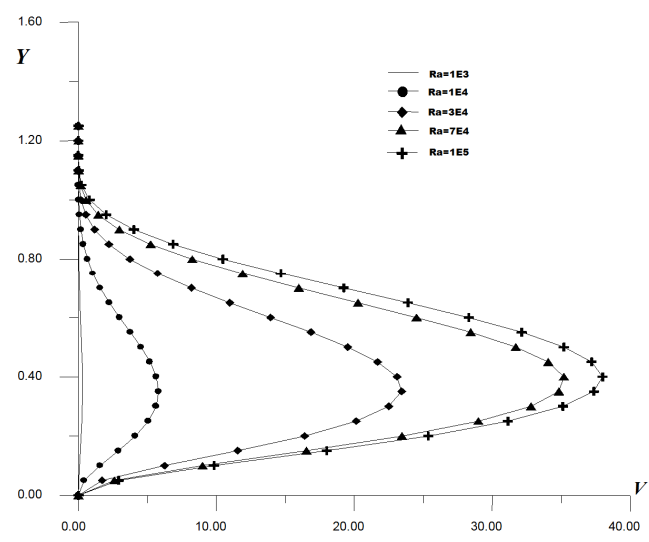

Fig. 6. Vertical velocity component profile at $\mathrm{X}=0.57$ (the middle) for $\mathrm{A}_{\mathrm{w}}=0.25$; $\mathrm{Ra}=10^{3} ; 10^{4} ; 3.10^{4} ; 7.10^{4} ; 10^{5}$

Figure 7 depicts the profiles of the velocity component along the bottom of the triangular part. Thus, powerful buoyancy forces disturb stagnant zones but one warms more than the other. The awakened fluid, in the corner being warmed, contributes to the convective effect aiding the opposite cell which sucks it up. The other cell is then reduced and takes a secondary extent. It can be seen that by increasing $\mathrm{Ra}$, the vertical velocity profiles lose their symmetry and attain high magnitudes in the central region. This high velocity moves warm air from the bottom following an oblique path toward the vicinity of the cold wall, where it undergoes deviation around each vortex area.

\subsubsection{Thermal Field}

Figure 8 represents the temperature profile along the bottom and Fig. 9 depicts the temperature profile at the middle. As it can be seen, the middle of the plate is more warmed. In this region, the temperature decreases with $\mathrm{Ra}$ values, but it remains highest at the plate. However, the recirculation zones enlarged by buoyancy forces mixes well the cold fluid and the arisen fluid 
from the bottom. We have to notice that, in a cavity still receiving a uniform heat flux, the bottom is not isothermal. This is in agreement with the thermal field structures illustrated in Fig. 10. For small Ra values $\left(R a \leq 10^{4}\right)$, the temperature distribution is almost the same as in the pure conduction case. However, for $\left(R a>10^{4}\right)$, the natural convection effect is dominant at the expense of conduction and a temperature inversion appears in the enclosure.
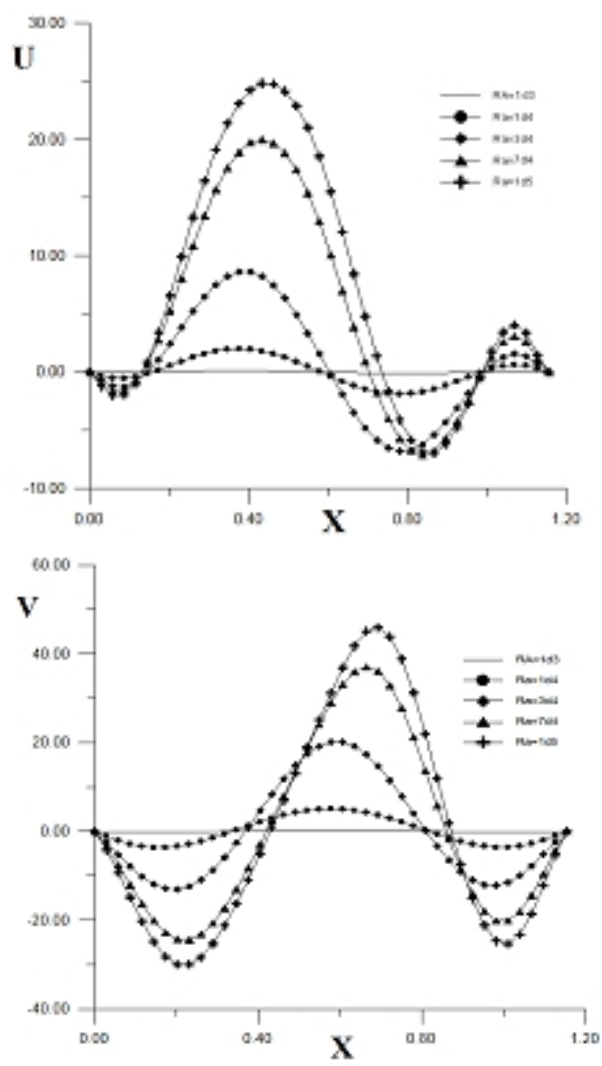

Fig. 7. Velocity components profiles along the bottom of the triangular part: horizontal component (up); vertical component (down).

\subsubsection{Local Nusselt Number}

As an example, the local Nusselt number variation across the inclined walls for an aspect ratio of 0.25 and different $\mathrm{Ra}$ values is shown in Fig. 11. As it can be noticed, the local Nusselt number increases to definite value at the intersection $(X=0$ and $X=1.14)$ of cold inclined walls and adiabatic walls which are heated more.

The high values of Nusselt number near the intersection give an indication that a given region within the neighborhood of this intersection accounts for more than a proportionate amount of heat transported across the inclined walls.

For a given $\mathrm{Ra}$, it can be seen that the Nusselt number admits a minimum at the upper summit $(X=0.57)$. This result had been expected because in this region the fluid is stagnant and there is no meaningful heat transfer across this section. Then, an increase in the amount of heat transfer is noticed when the Nusselt number reaches a secondary maxima around $X=0.28$ and $X=0.89$ that correspond to the mid-heights of the two inclined walls. This is the region reached by the heated fluid pushed upward from the bottom.

\subsection{Aspect Ratio Effect}

To study the effect of the aspect ratio on the flow structure and thermal field, we have increased $A_{w}=W / H$ from 0.25 to reach the value of 0.5 .

Figure 12 represents series of streamlines patterns for Ra of $10^{3}, 10^{4}, 10^{5}$ and $10^{6}$, Prandtl number of 0.71 , and dimension ratios of 0.25 and 0.5 . As it can be seen, for small Rayleigh number ( $R a \leq 10^{4}$ ), the streamlines patterns are almost the same for the two aspect ratios: two counter-rotating vortices are present in the enclosure and the eye of each vortex is located at the center of the half of the cross-section. However, the fluid volume becomes more important by increasing the cavity aspect ratio and the two cells grow in size. As the $\mathrm{Ra}$ is increased $\left(R a=10^{5}\right)$, the eye of each vortex moves towards to the right adiabatic wall for $A_{w}=0.25$, but the two cells remain near the bottom. However, for $A_{w}=0.5$, the left cell becomes the main vortex of high strength and large size. The right one becomes a secondary vortex of small size located near the top corner of the enclosure. This increase in Ra causes more strong cross-sectional flows. Further increase in the value of $\mathrm{Ra}\left(R a=10^{6}\right)$ causes secondary vortex to develop on the left corner of the enlarged enclosure $\left(A_{w}=0.5\right)$. Due to the large value of the $\mathrm{Ra}$ and the increasing of the aspect ratio, the structure of the flow is not symmetrical and justifies the opting for the computation of the entire physical domain. The newlydeveloped secondary vortex pushes the eye of the primary vortex further towards the right vertical wall. The cells' multiplicity homogenizes the thermal field by warming the core of the cavity. Obviously, the thermal field is sensitive to the fluid structure change such as shown by the series of isotherms patterns in Fig. 13.

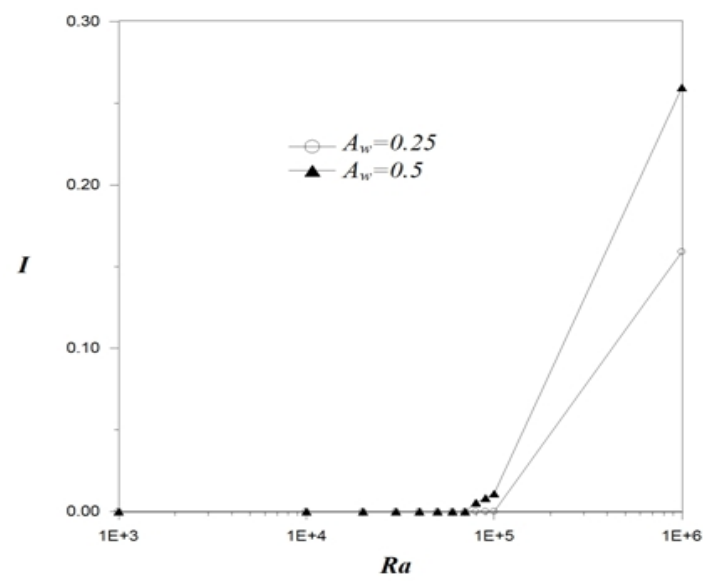

Fig. 14. Strength of asymmetry versus Ra for different aspect ratios

To monitor the strength of the asymmetry for different values of $\mathrm{Ra}$ (Fig. 14), we have determined the following integral: 


$$
I=\sqrt{\frac{\iint_{A}[\theta(X, Y)-\theta(-X, Y)]^{2} d X d Y}{\iint_{A}[\theta(X, Y)+\theta(-X, Y)]^{2} d X d Y}}
$$

Thus a purely symmetric flow yields $I=0$. It has been found that the critical value of $\mathrm{Ra}$, at which the symmetric flow regime no longer remains stable $(I \neq 0)$, depends on the value of the aspect ratio. Indeed, for $A_{w}=0.25$, this value is $\mathrm{Ra}=10^{5}$ whereas for $A_{w}=0.5$ the corresponding value is $\mathrm{Ra}=7.10^{4}$. As it can be seen in Fig. 14, the value of $I$ decreases as Ra goes down and the strength of asymmetry is more pronounced for $A_{w}=0.5$.

\section{Conclusion}

This paper has reported numerical results for steady, laminar, two-dimensional natural convection in a prismatic cavity with isothermal upper sidewalls, adiabatic vertical walls and receiving a uniform continuous heat flux at the bottom. The results presented show that the cavity's aspect ratio has a profound influence on the temperature and flow fields. On the other hand, the effect of small $\mathrm{Ra}$ values $\left(R a \leq 10^{4}\right)$ is not significant. Two counter-rotating vortices are present in the enclosure and the eye of each vortex is located at center of the half of the crosssection. As $\mathrm{Ra}$ is increased, the eye of each vortex moves towards the right vertical wall for $A_{w}=0.25$, but the two cells remain near the bottom. As for $A_{w}=0.5$, the left cell becomes the main vortex of high strength and large size. The right one becomes a secondary vortex of small size located near the top corner of the enclosure. This increase in $\mathrm{Ra}$ causes more strong cross-sectional flows. Further increase in $\mathrm{Ra}$ ( $R a=10^{6}$ ) causes secondary vortex to develop on the left corner of the enlarged enclosure $\left(A_{w}=0.5\right)$.

It has been found that a considerable proportion of the heat transfer across the inclined walls of the enclosure takes place near the intersection of the adiabatic vertical walls and cold inclined walls. Also, it has been noticed that, in a cavity still receiving a uniform heat flux, the bottom is not isothermal and the flow structure is sensitive to the cavity's shape. Many recirculation zones can occur in the core of the cavity and the heat transfer is dependent on the flow structure.

\section{ACKNOWLEDGEMENTS}

The authors would like to express their deepest gratitude to Mr Ali AMRI and his institution "The English Polisher" for their meticulous and painstaking review of the English text of the present paper.

\section{REFERENCES}

Asan, H. and L. Namli (2000). Laminar natural convection in a pitched roof of triangular cross section: summer day boundary condition. Energy and Buildings 33, 69-73.

Asan, H. and L. Namli (2001). Numerical simulation of buoyant flow in a roof of triangular cross section under winter day boundary conditions. Energy Build 33, 753-757.

Baliga, B.R. (1978). A Control-volume Based Finite Element Method for Convective Heat and Mass Transfer. PhD Thesis, University of Minnesota, Minneapolis, U.S.A.

Flack, R.D. (1979). Velocity measurements in two natural convection air flows using a laser velocimeter. J. Heat Transfer 101, 256-260.

Flack, R.D. (1980). The experimental measurement of natural convection heat transfer in triangular enclosures heated or cooled from below. J. Heat Transfer 102, 770-772.

Hajri, I., A. Omri and S. Ben Nasrallah (2007). A numerical model for the simulation of doublediffusive natural convection in a triangular cavity using equal order and control volume based on the finite element method. Desalination 206, 579-588.

Hakan, F.O., Y. Varol and A. Koca (2007). Laminar natural convection heat transfer in a shed roof with or without eave for summer season. Applied Thermal Engineering 27, 2252-2265.

Holtzman, G.A., R.W. Hill and K.S. Ball (2000). Laminar natural convection in isosceles triangular enclosures heated from below and symmetrically cooled from above. J. Heat Transfer 122, 485491.

Karyakin, Y.E. (1989). Transient natural convection in prismatic enclosures of arbitrary cross section. Int. J. Heat Mass Transfer 32(6), 1095-1103.

Koca, A., F.O. Hakan and Y. Varol (2007). The effects of Prandtl number on natural convection in triangular enclosures with localized heating from below. International Communications in Heat and Mass Transfer 34, 511-519.

Omri, A. (2000). Etude de la convection mixte à travers une cavité par la méthode des volumes de contrôle à base d'éléments finis. Thèse de Doctorat, Faculté des Sciences de Tunis, pp. 1-184.

Omri, A, J. Orfi and S. Ben Nasrallah (2005). Natural convection effects in solar stills. Desalination 183, 173-178.

Ridouane, E.H. and A. Campo (2005). Experimentalbased correlations for the characterization of free convection of air inside isosceles triangular cavities with variable apex angles. Experimental Heat Transfer 18, 81- 86.

Tzeng, S.C., J.H. Liou and R.Y. Jou (2005). Numerical simulation-aided parametric analysis of natural convection in a roof of triangular enclosures. Heat Trans. Eng. 26, 69-79. 
Varol, Y., A. Koca and H.F. Oztop (2007). Natural convection heat transfer in Gambrel Roofs. Building and Environment 42, 1291-1297.

Varol, Y., F.O. Hakan and T. Yilmaz (2007). Natural convection in triangular enclosures with protruding isothermal heater. International Journal of Heat and Mass Transfer 50, 24512462.
Volker, V., T. Burton and S.P. Vanka (1989). Finite Volume Multigrid Calculation of Natural Convection Flows On Unstructured Grid. Numerical Heat Transfer (Part B) 30,1-22.
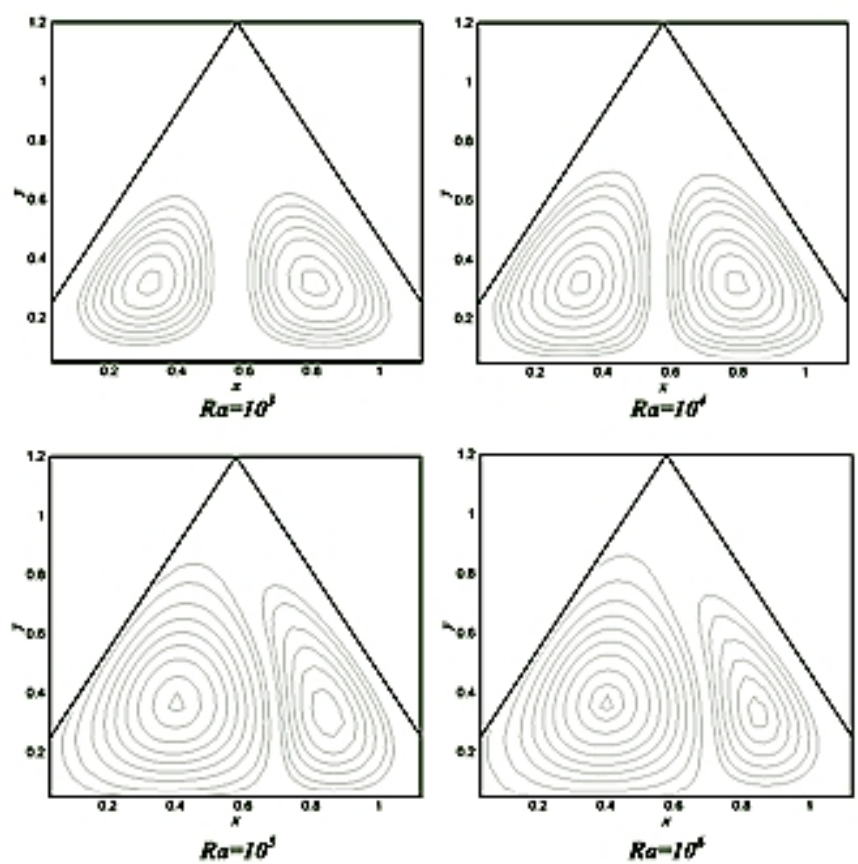

Fig. 5. Streamlines for $A_{w}=0.25 ; R a=10^{3} ; 10^{4} ; 10^{5} ; 10^{6}$

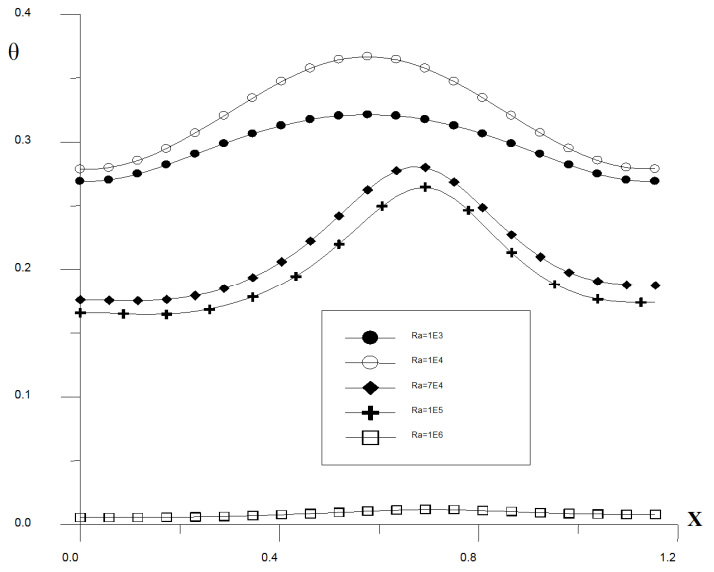

Fig. 8. Temperature profile at the bottom for $\mathrm{A}_{\mathrm{w}}=0.25, \mathrm{Ra}=10^{3} ; 10^{4} ; 7.10^{4} ; 10^{5} ; 10^{6}$

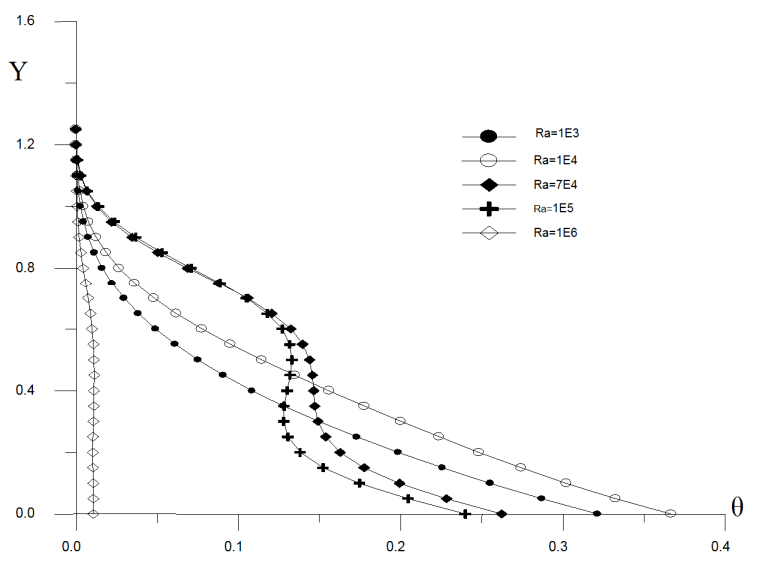

Fig. 9. Temperature profile at the middle for $\mathrm{A}_{\mathrm{w}}=0.25, \mathrm{Ra}=10^{3} ; 10^{4} ; 7.10^{4} ; 10^{5} ; 10^{6}$ 


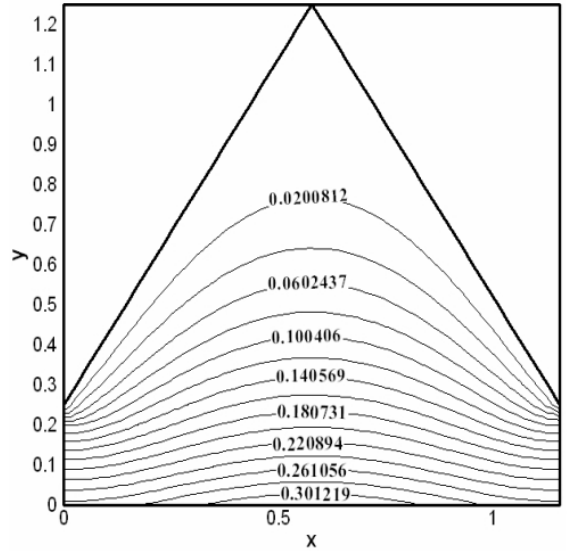

$\mathbf{R a}=10^{3}$

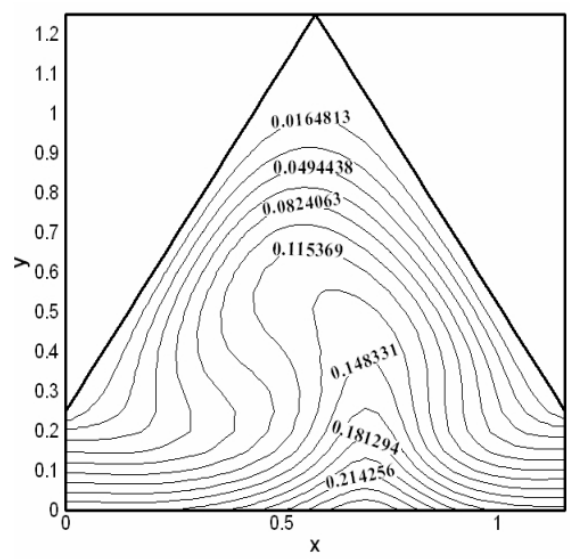

$\mathbf{R a}=10^{5}$

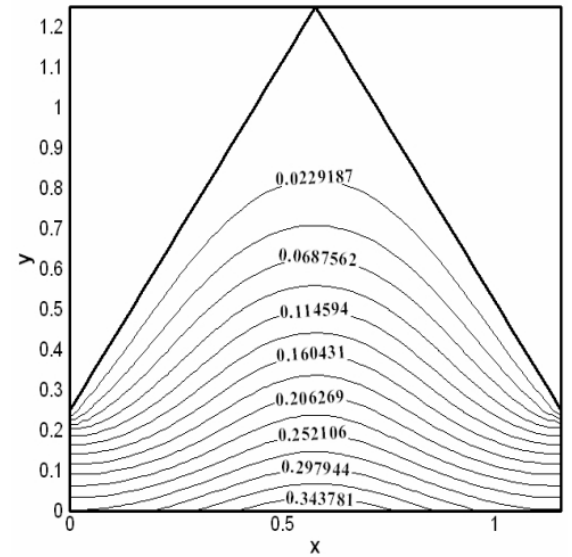

$\mathrm{Ra}=10^{4}$

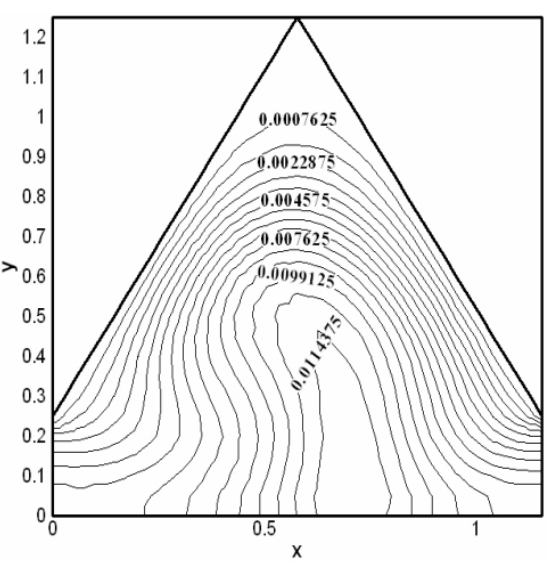

$\mathrm{Ra}=10^{6}$

Fig. 10. Thermal field for $A_{w}=0.25$ and different Rayleigh number values
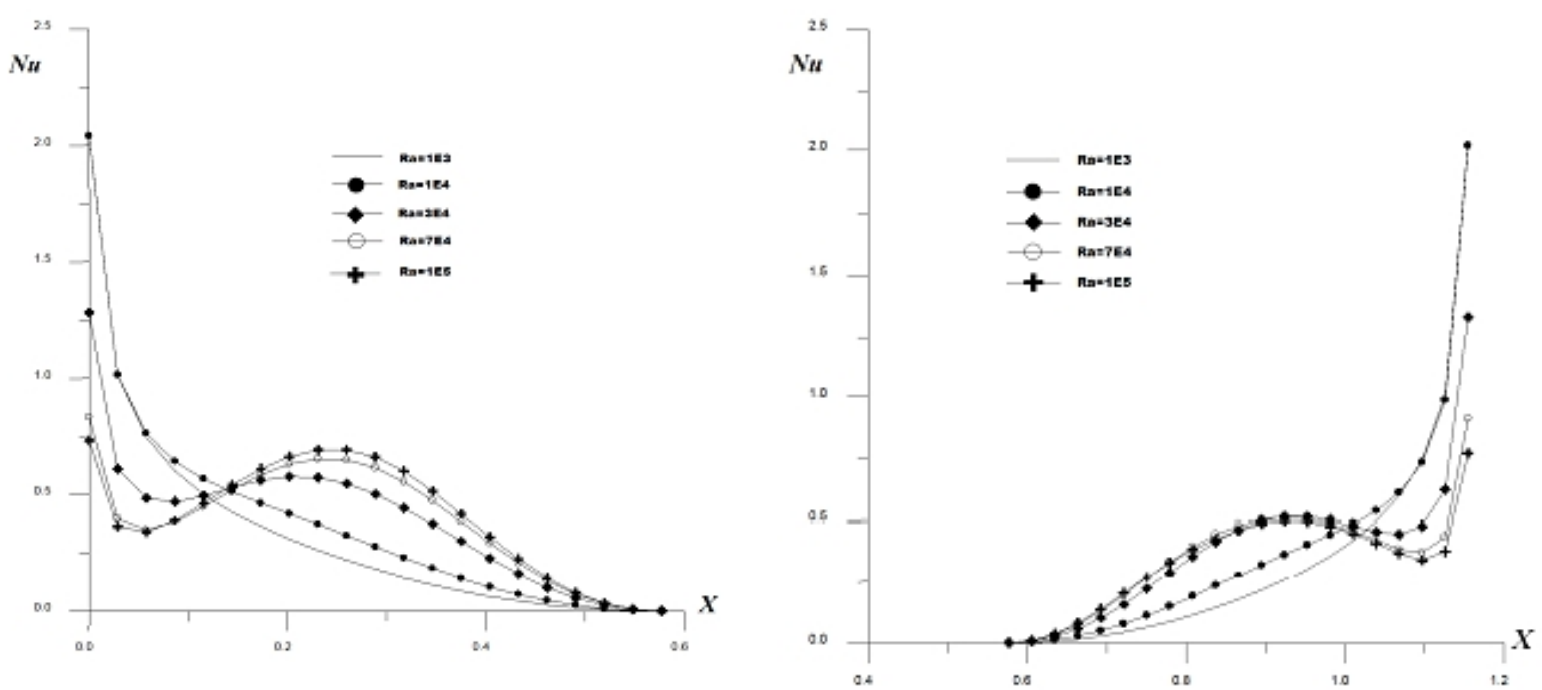

Fig. 11. Local Nusselt number versus $X$ for different Rayleigh number values: left inclined wall (left) and right inclined wall (right) 

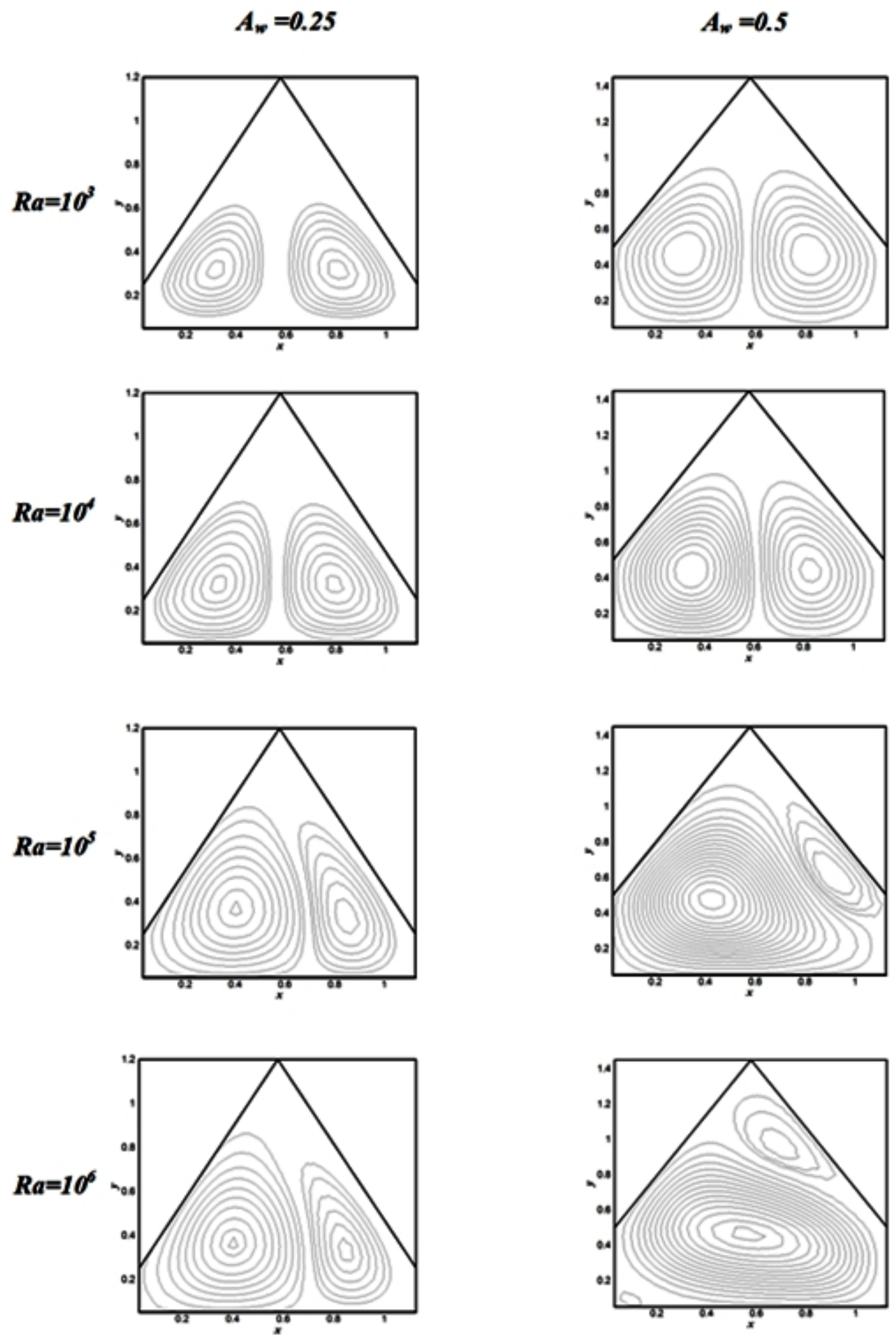

Fig. 12. Streamlines for different aspect ratios and different Rayleigh number values 
A. Walid and O. Ahmed / JAFM, Vol. 3, No. 2, pp. 77-86, 2010.
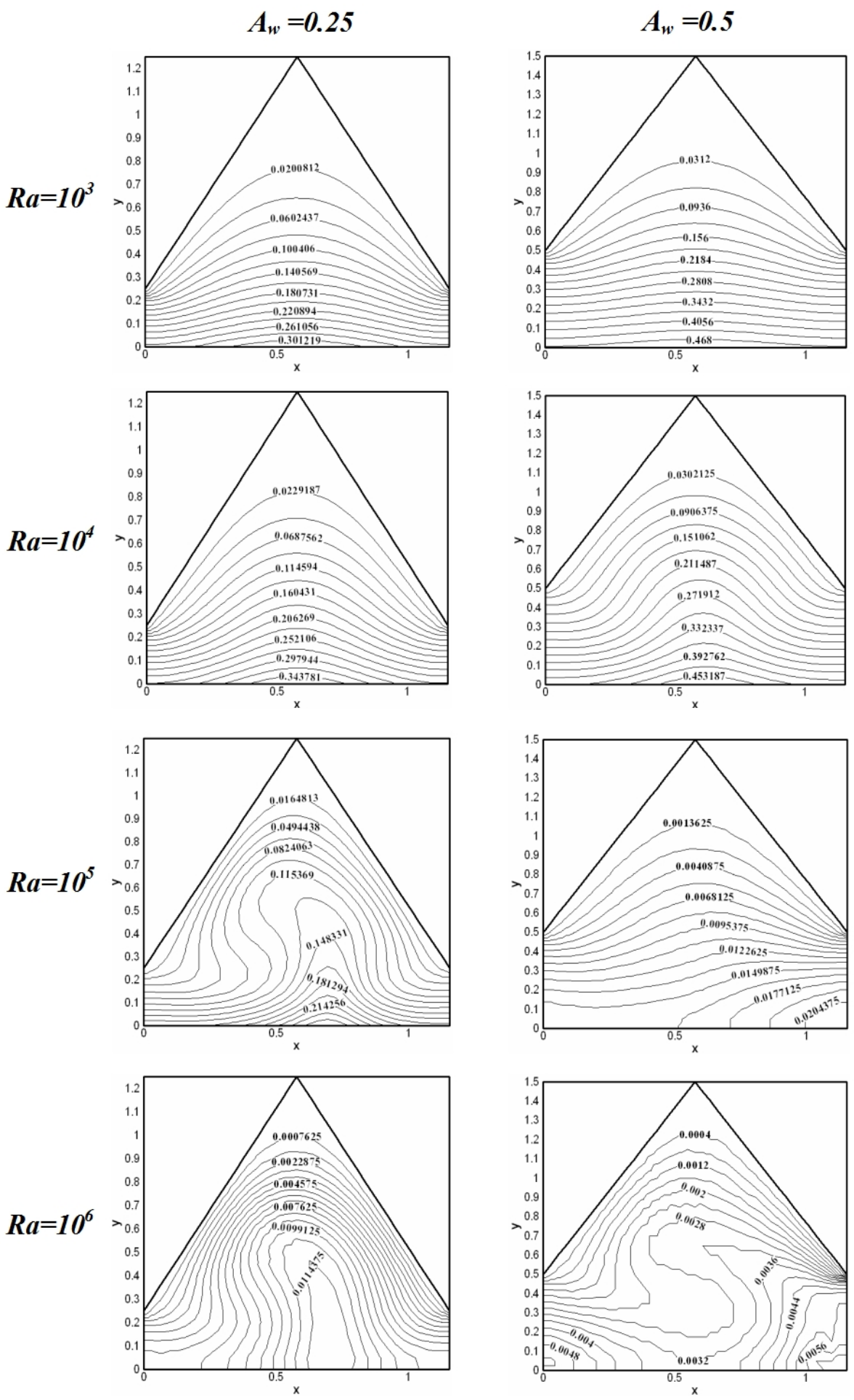

Fig. 13. Isotherms patterns for different aspect ratios and different Rayleigh number values 